\title{
An Assessment of Saudi Hospitals’ Disaster Preparedness
}

\author{
Majed Mohammed Alsalem ${ }^{1 *}$, Saad Abdullah Alghanim ${ }^{2}$
}

\author{
${ }^{1}$ Hospital Administration, King Saud University, SAUDI ARABIA \\ ${ }^{2}$ Business Administration, King Saud University, SAUDI ARABIA \\ *Corresponding Author: timesa@gmail.com
}

Citation: Alsalem, M. M. and Alghanim, S. A. (2021). An Assessment of Saudi Hospitals' Disaster Preparedness. European Journal of Environment and Public Health, 5(2), em0071. https://doi.org/10.21601/ejeph/9663

ARTICLE INFO

Received: 21 Aug. 2020

Accepted: 10 Nov. 2020

\begin{abstract}
Objective: The study aims to assess the level of Saudi Hospital Disaster Preparedness (HDP). Methods: This study has utilized exploratory quantitative design with a structured self-administered questionnaire. It has adopted a convenient sampling technique, which has included nine hospitals within three Saudi Cities (Riyadh, Jeddah, and Dammam). The questionnaire contains 48 indicators in six HDP domains: planning, structural, non-structural, management, functional, and human resource competency capacities. Results: The level of Saudi HDP was $69.8 \%$. The domain of management capacity had the highest level of preparedness, at $83.6 \%$, while human resources competency capacity was the least prepared, at $63 \%$. The Spearman correlation revealed a positive significant relationship between preparing to respond to a disaster when occur and all HDP capacity indicators, $r(46)=0.424, p=<.001$. A one-way ANOVA test showed a significant difference in HDP between the three cities; Riyadh hospitals were the most prepared, $F(2,313)=4.343, p<.05$. It also showed that there were significant differences in preparedness between the $\mathrm{MOH}$, other governmental, and university hospitals; university hospitals were the most prepared, $F(2,313)=5.087, p<.05$. Conclusion: Overall, Saudi hospitals have a high level of HDP, which indicates that they are likely to function well in the eventuality of disasters. There is a great opportunity to strengthen HDP capacities to improve hospital readiness in the medium-long term. The implemented HDP assessment tool should be built into hospitals' systems and coordinated among involved governmental sectors.
\end{abstract}

Keywords: disaster, hospital, preparedness, capacity

\section{INTRODUCTION}

Disasters have posed fatal consequences to many countries including Saudi Arabia, which possesses compounding factors such as vast geographical territory, extreme weather fluctuations, mass gatherings (Haji and Umra), terrorist attacks, and epidemics that could intensify disasters' consequences. In the time of disasters, hospitals could bear the heaviest consequences, as a large number of patients may be admitted and hospitals host the most vulnerable populations and very expensive equipment. Hospitals are considered the main components in healthcare systems in providing essential medical care to the community, particularly during disasters (Mulyasari et al., 2013; WHO, 2009).

Disasters cause hundreds of injured people who are in desperate need for hospitals treatment, which subsequently lead to overwhelming or crippling the capacity of hospitals, especially when hospitals are not well-prepared to respond to such a massive influx of casualties (Bayntun et al., 2012). Thus, deficiency in hospital preparedness in facing disasters would potentially result in rapid spread of diseases, or loss of lives and properties leaving little time to implement ad-hoc mitigation measures (Kapur and Smith, 2010; Chaffee and Oster, 2005). This deficiency could be attributed to the deficit in HDP capacities including planning, management, structural, non-structural, functional, and human competency (Mulyasari et al., 2013; Kapur and Smith, 2010).

Hence, this study aims to assess these capacities, provide an overall assessment of Saudi HDP, and define the differences between the hospitals' types; Ministry of Health (MOH), other governmental, and university hospitals as well as the differences between hospitals in three cities; Riyadh, Jeddah, and Dammam. This study will include the study methodology, results, discussion and conclusion to achieve its goal.

\section{METHODOLOGY}

The study methodology aids in describing, explaining and assessing HDP in Saudi cities. It includes the study design, sampling, data collection, and data analysis. 
Table 1. Level of HDP according to HIS

\begin{tabular}{lll}
\hline $0 \%-35 \%$ & C & $\begin{array}{l}\text { Urgent intervention measures are needed. The hospital's current safety levels are inadequate to protect the lives of } \\
\text { patients and hospital staff during and after a disaster. }\end{array}$ \\
\hline $36 \%-65 \%$ & B & $\begin{array}{l}\text { Intervention measures are needed in the short-term. The hospital's current safety levels are such that patients, } \\
\text { hospital staff, and its ability to function during and after a disaster are potentially at risk }\end{array}$ \\
\hline $66 \%-100 \%$ & A & $\begin{array}{l}\text { It is likely that the hospital will function in case of a disaster. It is recommended, however, to continue with measures } \\
\text { to improve response capacity and to carry out preventive measures in the medium- and long-term to improve the } \\
\text { safety level in case of disaster }\end{array}$ \\
\hline
\end{tabular}

Source: (WHO, 2015)

\section{Study Design}

The study design is based on a descriptive quantitative technique that aims to answer the research questions concerning HDP in Saudi Cities. The study has obtained data by utilizing a cross-sectional self-administered questionnaire that was constructed based on the literature review and pilot study. Moreover, the assessment tool has been structured based on literature review, which has revealed that there are many HDP tools and checklists with different scopes and magnitudes, but all of these studies have agreed on the fact that there is no consensus exists on standardized, comprehensive, and validated instrument to assess HDP for all hazards (Heidaranlu, Ebadi, et al., 2017; Heidaranlu et al., 2015; Jenkins et al., 2009; Kaji et al., 2008; Nekoie-Moghadam et al., 2016). Therefore, this study has utilized Hospital Safety Index (HIS) assessment criteria, which was develop by World Health Organization (WHO) in order to define the level of preparedness for each indicator. It utilizes three scales; low, average, and high, with a maximum value of $100 \%$ to be achieved in all variables. The end result is classified in to level A, B, or C as Table 1 (WHO, 2015).

\section{Sampling}

The study has targeted hospitals in three cities that are considered the major cities in Saudi Arabia, namely; Riyadh, Jeddah, and Dammam. A convenience sampling method has been implemented to include nine hospitals among three types of hospitals; $\mathrm{MOH}$, University, and other governmental hospitals. These types of hospitals provide $80 \%$ of healthcare services in country. Within these hospitals, a convenience sampling method also has been applied for participants in six healthcare professionals categories; infection control, emergency, safety, maintenance, quality, and management due to their direct involvement in hospital disaster management.

\section{Data Collection}

The survey was conducted between November 2018 and February 2019 after obtaining the ethical approval from the Institutional Review Board (IRB) held on 30/10/2018 with reference No.: KSU-HE-18-22. 450 questionnaires (hard and electronic copies) were distributed to all hospitals and 364 return. Forty-eight (48) of them were excluded due to the incompleteness of demographic or general information about the hospital and the incompleteness of the indicators' assessment. The overall response rate was $70.2 \%$ with three hundred and sixteen (316) completed questionnaires that have been incorporated into software IBM SPSS version 20.0 for analysis. The questionnaire contained three parts. First, it contained demographic and social characteristics of the participants. Second, it contained general information about the selected hospitals concerning their cities, their types, and the number of disasters that face these hospitals. Third, it consisted of 48 questions and statements to assess the level of HDP in six main capacities categories; (1) planning (2) management (3) structural (4) non-structural (5) functional (6) human resource competency. The distribution of questionnaires and data collection was self-administered by the researcher after obtaining approval from Academic Affairs departments in the designated hospitals.

\section{Data Analysis}

Data management and statistical analysis were done using software IBM SPSS version 20.0. Several statistical tools were applied including means, standard division, frequencies, and percentages to analyzing the demographic characteristics of the participants and the general information about the hospitals. They were also utilized to define the level of each indicator to define the level of preparedness on the level each main category and the overall HDP. The reliability test of Cronbach's alpha has achieved 97\% and the face validity and content validity of the survey was previously assessed and approved by six experts in the field of disaster management. The 48 statements were assessed on five level scale where (1) represent very low and (5) very high. An assessment criterion that was established by the WHO and adopted in the Hospital Safety Index (HSI) has been utilized as a guideline to judge the level of preparedness for each indicator. Proportions of indicators were calculated and preparedness was classified as High (= 66\% $-100 \%)$, Medium (36-65\%) or Low (= 35\%-0\%) based on the actual range of the indicators (Ardalan et al., 2014; Sunindijo et al., 2019; WHO, 2016, 2019, 2012).

\section{RESULTS}

\section{Demographic Characteristics}

The study has included nine hospitals in three cities in Saudi Arabia. 173 respondents were working in hospitals located in Riyadh city, 71 respondents in Dammam city, and 72 in Jeddah. 37\% (117) of the respondents were working in $\mathrm{MOH}$ hospitals, $46.2 \%$ in other governmental hospitals (146 respondents), and 53 respondents representing $16.7 \%$ in University hospitals. $19 \%$ of respondents believed that their hospital was not prepared for disasters, and $81 \%$ believed they were well prepared. The total number of participants was 316 out of 450 . The majority of the respondents were males with $63.3 \%$, while the females represented $36.7 \%$ of the total sample size. The results also indicated that the average mean of age was 38.725 years old. The respondents' experiences in disaster 
Table 2. Demographic Characteristics

\begin{tabular}{cccc}
\hline \multirow{4}{*}{ Cities } & Variables & No. & \% \\
\cline { 2 - 4 } & Riyadh & 173 & 54.7 \\
\cline { 2 - 4 } & Dammam & 71 & 22.5 \\
\hline \multirow{3}{*}{ Type of hospitals } & Jeddah & 72 & 22.8 \\
\cline { 2 - 4 } & MOH & 117 & 37 \\
\cline { 2 - 4 } & Other Gov. & 146 & 46.2 \\
\hline \multirow{2}{*}{ Respond to disasters } & University & 53 & 16.8 \\
\cline { 2 - 4 } & No & 60 & 19 \\
\hline \multirow{2}{*}{ Gender } & Yes & 256 & 81 \\
\cline { 2 - 4 } & Female & 116 & 36.7 \\
\hline \multirow{3}{*}{ Age } & Male & 200 & 63.3 \\
\cline { 2 - 4 } & From 23 to 35 & 135 & 42.7 \\
\cline { 2 - 4 } Experience & From 36 to 47 & 120 & 38 \\
\cline { 2 - 4 } & 48 and more & 61 & 19.3 \\
\cline { 2 - 4 } & From 1 to 13 & 186 & 58.9 \\
\hline \multirow{3}{*}{ Department } & From 14 to 26 & 130 & 41.1 \\
\cline { 2 - 4 } & Infection control & 36 & 11.4 \\
\cline { 2 - 4 } & Emergency & 98 & 31 \\
\cline { 2 - 4 } & Quality & 64 & 20.3 \\
\cline { 2 - 4 } & Safety & 44 & 13.9 \\
\cline { 2 - 4 } & Maintenance & 29 & 9.2 \\
\cline { 2 - 4 } & Management & 45 & 14.2 \\
\cline { 2 - 4 } & &
\end{tabular}

Total respondents $=316$

Table 3. Assessment of HDP Capacities

\begin{tabular}{ccc}
\hline HDP Indicators & Percentage & Assessment Level \\
\hline Planning & $69 \%$ & High \\
\hline Management & $83.6 \%$ & High \\
\hline Structural & $68.7 \%$ & High \\
\hline Non- Structural & $69.9 \%$ & High \\
\hline Functional & $65 \%$ & Medium \\
\hline Human Resources Competency & $63 \%$ & Medium \\
\hline Overall assessment & $69.8 \%$ & High \\
\hline
\end{tabular}

management were ranging from 1 to 13 years representing $58.9 \%$ of the total respondents, while $41.1 \%$ had experience above 14 years. The overall mean of experiences was 12.798 years. The majority of respondents was working in emergency department and representing $31 \%$, while $20.3 \%$ of them in quality, $14.2 \%$ in management positions, $13.9 \%$ in safety, $11.4 \%$ in infection control, and $9.2 \%$ in maintenance.

\section{Assessment of HDP Capacities}

The overall level of Saudi HDP was 69.8\%. The level of HDP capacity for human resource competency was $63 \%$, functional
Table 5. Relationship at the level of All Indicators

\begin{tabular}{ccc}
\hline \multirow{2}{*}{ HDP indicators } & Correlation Coefficient & $.424^{* * *}$ \\
\cline { 2 - 3 } & Sig. (2-tailed) & .000 \\
\hline
\end{tabular}

$65 \%$, structural $68.7 \%$, planning $69 \%$, non-structural $69.9 \%$, and management $83.6 \%$.

\section{Relationships between Participants' Experience Regarding their Hospital Preparedness and the Assessment of the Six Capacity Indicators}

Table 4 shows that participants' experience regarding their hospital preparedness level to respond to disasters correlated significantly with: planning, management, structural, non-structural, functional, and human competency capacities, with spearman correlation values: $0.455,0.419$, $0.267,0.264,0.398$, and 0.335 respectively.

The Spearman correlation in Table 5 revealed a positive significant relationship between preparing to respond to a possible disaster when occur and all HDP capacity indicators, $r$ $(46)=0.424, p=<.001$. Since the value near from 1 , the relationship is defined as a strong relationship.

\section{Differences in HDP between Riyadh, Jeddah, and Dammam Cities}

To identify the differences in hospital disasters preparedness between Riyadh, Jeddah, and Dammam cities' analysis of variance was used, as shown in Table 6 . The results indicated that the mean of HDP in Riyadh city was 186.14 degree, the mean of HDP in Jeddah city was 174.61 degree, and the mean of HDP in Dammam city was 184.52 degree. These results represented some differences in means of HDP according to cities.

To determine the direction of this difference, an LSD test was performed as in Table 7, from the table the mean difference between Riyadh and Jeddah 11.53340 with sig. 0.004 , the mean difference between Riyadh and Dammam 1.62338 with sig. 0.684 , the mean difference between Dammam and Jeddah 9.91002 with sig. 0.037, these results indicate that the difference in HDP was in favor of Riyadh.

A one-way ANOVA test in Table 8 showed that there was a significant difference in disaster preparedness between the hospitals in the different cities; hospitals in Riyadh city were the most prepared, $F(2,313)=4.343, p<.05$.

Table 4. Relationship at the level of Main Six Indicators' Categories

\begin{tabular}{|c|c|c|c|c|c|c|c|}
\hline & & Planning & Mgmt & Structural & Non structural & Functional & $\begin{array}{c}\text { Human } \\
\text { competency }\end{array}$ \\
\hline Prepare to respond & spearman Correlation & $.455^{* * *}$ & $.419 * *$ & $.267^{* * * *}$ & $.264^{* * * *}$ & $.398 * *$ & $.335^{* * *}$ \\
\hline to disaster & Sig. (2-tailed) & .000 & .000 & .000 & .000 & .000 & .000 \\
\hline
\end{tabular}

Table 6. Differences in HDP between Riyadh, Jeddah, and Dammam cities

\begin{tabular}{|c|c|c|c|c|c|c|c|c|}
\hline \multirow{2}{*}{ Cities } & \multirow{2}{*}{$\mathbf{N}$} & \multirow{2}{*}{ Mean } & \multirow{2}{*}{$\begin{array}{c}\text { Std. } \\
\text { Deviation }\end{array}$} & \multirow{2}{*}{ Std. Error } & \multicolumn{2}{|c|}{ 95\% Confidence Interval for Mean } & \multirow{2}{*}{ Mini } & \multirow{2}{*}{ Max. } \\
\hline & & & & & Lower Bound & Upper Bound & & \\
\hline Riyadh & 173 & 186.14 & 27.46 & 2.08 & 182.02 & 190.26 & 62 & 228 \\
\hline Jeddah & 72 & 174.61 & 37.23 & 4.38 & 165.86 & 183.36 & 71 & 228 \\
\hline Dammam & 71 & 184.52 & 17.59 & 2.08 & 180.35 & 188.68 & 138 & 228 \\
\hline Total & 316 & 183.15 & 28.55 & 1.60 & 179.99 & 186.31 & 62 & 228 \\
\hline
\end{tabular}


Table 7. LSD Test according to cities

\begin{tabular}{|c|c|c|c|c|c|c|}
\hline \multirow{2}{*}{ (I) City } & \multirow{2}{*}{ (J) City } & \multirow{2}{*}{ Mean Difference (I-J) } & \multirow{2}{*}{ Std. Error } & \multirow{2}{*}{ Sig. } & \multicolumn{2}{|c|}{ 95\% Confidence Interval } \\
\hline & & & & & Lower Bound & Upper Bound \\
\hline \multirow{2}{*}{ Riyadh } & Jeddah & $11.53340^{*}$ & 3.96262 & .004 & 3.7367 & 19.3301 \\
\hline & Dammam & 1.62338 & 3.98227 & .684 & $-6.2120-$ & 9.4588 \\
\hline \multirow{2}{*}{ Jeddah } & Riyadh & $-11.53340-^{*}$ & 3.96262 & .004 & $-19.3301-$ & $-3.7367-$ \\
\hline & Dammam & $-9.91002-^{*}$ & 4.72565 & .037 & $-19.2081-$ & $-.6120-$ \\
\hline \multirow{2}{*}{ Dammam } & Riyadh & $-1.62338-$ & 3.98227 & .684 & $-9.4588-$ & 6.2120 \\
\hline & Jeddah & $9.91002^{*}$ & 4.72565 & .037 & .6120 & 19.2081 \\
\hline
\end{tabular}

* The mean difference is significant at the 0.05 level.

Table 8. One way ANOVA test according to cities

\begin{tabular}{|c|c|c|c|c|c|}
\hline & Sum of Squares & Df & Mean Square & $\mathbf{F}$ & Sig. \\
\hline Between Groups & 6934.49 & 2 & 3467.24 & & \\
\hline Within Groups & 249874.21 & 313 & 798.32 & 4.343 & .014 \\
\hline Total & 256808.70 & 315 & & & \\
\hline
\end{tabular}

Table 9. Type of hospitals Descriptive statistics

\begin{tabular}{|c|c|c|c|c|c|c|c|c|}
\hline \multirow{2}{*}{ Type of hospitals } & \multirow{2}{*}{$\mathbf{N}$} & \multirow{2}{*}{ Mean } & \multirow{2}{*}{$\begin{array}{c}\text { Std. } \\
\text { Deviation }\end{array}$} & \multirow{2}{*}{ Std. Error } & \multicolumn{2}{|c|}{ 95\% Confidence Interval for Mean } & \multirow{2}{*}{ Mini. } & \multirow{2}{*}{ Max. } \\
\hline & & & & & Lower Bound & Upper Bound & & \\
\hline $\mathrm{MOH}$ & 117 & 178.08 & 32.30 & 2.98 & 172.17 & 184 & 62 & 228 \\
\hline Other Gov. & 146 & 183.67 & 25.25 & 2.08 & 179.54 & 187.80 & 100 & 228 \\
\hline University & 53 & 192.90 & 26.02 & 3.57 & 185.73 & 200.07 & 84 & 228 \\
\hline Total & 316 & 183.15 & 28.55 & 1.60 & 179.99 & 186.31 & 62 & 228 \\
\hline
\end{tabular}

Table 10. LSD Test for HDP according to types of hospitals

\begin{tabular}{ccccccc}
\hline $\begin{array}{c}\text { (I) type of } \\
\text { hospital }\end{array}$ & $\begin{array}{c}\text { (J) type of } \\
\text { hospital }\end{array}$ & Mean Difference (I-J) & Std. Error & Sig. & \multicolumn{2}{c}{ 95\% Confidence Interval } \\
\cline { 2 - 7 } & Lother Gov. & $-5.5857-$ & 3.4978 & .111 & $-12.467-$ & 1.296 \\
\cline { 2 - 7 } & University & $-14.8201-^{*}$ & 4.6674 & .002 & $-24.003-$ & $-5.636-$ \\
\hline \multirow{2}{*}{ Other Gov. } & MOH & 5.5857 & 3.4978 & .111 & $-1.296-$ & 12.467 \\
\cline { 2 - 7 } & University & $-9.2344-^{*}$ & 4.5206 & .042 & $-18.129-$ & $-.3398-$ \\
\hline \multirow{2}{*}{ University } & MOH & $14.8201^{*}$ & 4.6674 & .002 & 5.6366 & 24.003 \\
\cline { 2 - 7 } & Other Gov. & $9.2344^{*}$ & 4.5206 & .042 & .3398 & 18.129 \\
\hline
\end{tabular}

* The mean difference is significant at the 0.05 level.

Table 11. One way ANOVA for Differences in HDP Between types of hospitals

\begin{tabular}{|c|c|c|c|c|c|}
\hline & Sum of Squares & Df & Mean Square & $\mathbf{F}$ & Sig. \\
\hline Between Groups & 8084.81 & 2 & 4042.40 & & \\
\hline Within Groups & 248723.89 & 313 & 794.64 & 5.087 & .007 \\
\hline Total & 256808.70 & 315 & - & & \\
\hline
\end{tabular}

Differences in HDP between MOH, Other Governmental, and University Hospitals

Table 9 shows some descriptive statistics for HDP in $\mathrm{MOH}$, Other Governmental, and University Hospitals, the results in the table indicated that the mean of HDP in $\mathrm{MOH}$ was 178.0855 degree, the mean of HDP in Other Governmental was 183.6712 degree, and the mean of HDP in University Hospitals was 192.9057 degree, that means there are Differences in means of HDP according to hospital type.

To determine the direction of this difference, an LSD test (Table 10) was performed as in Table 13, from the table the mean difference between University Hospital and $\mathrm{MOH}$ hospital 14.82019 with sig. 0.002 , the mean difference between University Hospital and Other Governmental Hospital 9.23443 with sig. 0.042, the mean difference between Other Governmental Hospital and MOH hospital 5.58576 with sig. 0.111 , these results indicate that the difference in HDP was in favor of University Hospital.
The one-way ANOVA test also in Table 11 showed that there were significant differences in preparedness between the $\mathrm{MOH}$, other governmental, and university hospitals; university hospitals were the most prepared, $F(2,313)=5.087$, $p<.05$.

\section{DISCUSSION}

Enhancement of HDP capacities should be initiated through the proper assessment of six main capacity categories that consisted of planning, management, structural, nonstructural, functional, and human resources competency. This assessment will aid in identifying the need to allow decisionmakers to formulate appropriate policies and procedures, determine priorities, allocate proper resources, and implement improvements to elevate the level of HDP when disasters occur. 
Forty- eight indicators under six main capacities were employed to assess the HDP in three Saudi cites namely; Riyadh, Jeddah, and Dammam. Our results showed that the overall level of HDP is $69.8 \%$ based on the respondents' assessments of the whole number of indicators within their hospitals. This level is considered a high level of HDP according to the WHO classification in its safety hospital index (SHI) for the category (A).

The level HDP management capacity in Saudi hospitals has achieved the highest level among the other main indicators with $83.6 \%$ assessment score, which similar to the results of several indictors in another study conducted in Makka city (AlShareef et al., 2017). Furthermore, non-structural capacity has achieved the second highest level among the other main capacities with $69.9 \%$ assessment score. This study has also found that there significant difference in non-structural capacity among all types investigated hospitals. This finding was supported by Bajow and AlKhalil's (2014) study where they found that there was a significant difference in the safety of lifeline systems, and architectural and furnishings among six hospitals in Jeddah city. Moreover, planning capacity has achieved a high level with 69\% assessment score. These results are supported by two studies conducted by Al-shareef et al. (2017) in 17 hospitals in Makka city, and by Shalhoub, Khan, and Alaska (2017) in private hospital in Riyadh city. Al-shareef et al. (2017) and this study results emphasized the importance of implementing the Hazard Vulnerability Analysis to develop their Emergency Operations Plan. In addition, structural capacity has achieved high level with $68.7 \%$ assessment score. Bajow and AlKhalil (2014) agreed with this results where they concluded that the level of HDP structural capacity is between Good and Very Good.

On the other hand, the functional capacity in Saudi hospitals has achieved the second lowest level among the other main capacities with $65 \%$ assessment score, which corresponds to the medium (B) category according to the WHO SHI. The study results of Bajow and Al-Khalil (2014) supported some of our results regarding the level of surge capacity and communication. They found that surge capacity and communication levels in Jeddah hospitals were to be good to very good preparedness levels. Finally, HDP human resources competency capacity in Saudi hospitals has achieved the lowest level among the other main capacities with $63 \%$ assessment score. Nofal et al. (2018) have found similar results at a university tertiary health care hospital in central Riyadh in which they concluded that the level of knowledge and awareness of healthcare professionals was satisfactory with a neutral level of attitude, practice, and familiarity regarding HDP. The study results are supported by three studies conducted by Alrazeeni (2015), Ibrahim (2014), and Althobaity et al. (2017). Alrazeeni (2015) who found that participants had weakly to moderate knowledge HDP level, and recommended to integrate HDP knowledge. Ibrahim (2014) surveyed 252 registered batches of bridging nursing students and found that there were lacking knowledge and practices with an average level of attitude and neutral familiarity with HDP. Althobaity et al. (2017) also supports this study results. They found a moderate level of knowledge among nurses in Saudi Arabia concerning HDP gained from disaster training and drills and added that nurses working in military hospitals had more knowledge than those $\mathrm{MOH}$ countries' human competency capacity level in HDP. Furthermore, Baker, Alamri, and Aboshaiqah (2019) have concluded that the knowledge of 350 nurses in five governmental hospitals in Medina achieved a satisfactory level with neutral level of involvement, preparedness, and commitment among them. AlHarastani et. al. (2020) confirmed the previous studies where they found that the overall knowledge level of hospital staff concerning HDP was satisfactory.

This study has concluded that there is a positive significant relationship between preparing to respond to a possible disaster when occur and all HDP capacity indicators, which indicates that applying general basic principles that is based on all-hazards approach will prepared hospitals in the eventuality of any type of disasters (CDC, 2013; White et al., 2012; WHO, 2015). In addition, this study has found that there was a significant difference in HDP between types and city of the hospitals, which is supported by Bajow and AlKhalil (2014) and Althobaity et al. (2017).

\section{CONCLUSION}

This study aimed to assess the overall level of HDP in Saudi hospitals through constructing a tool that contained six main HDP capacities. 316 healthcare professionals from nine hospitals responded to the survey to assess Saudi HDP in six main capacities. These capacities which contained planning, management, structural, non-structural, functional, and human resources competency have scored 69\%, 83.6\%, 68.7\%, $69.9 \%, 65 \%$, and $63 \%$ respectively. The overall level of Saudi HDP achieved a high level of HDP (69.8\%) comparing to the WHO criteria. It indicated that Saudi hospitals are likely to function when disasters occur. Furthermore, there is a great opportunity to strengthen our HDP capacities to improve hospitals' readiness and to implement protective measures in the medium- and long-term to improve the safety level when disasters occur. The human resources competency capacity was the lowest capacity among the others, which necessitate the importance to enhance the awareness, education, and training among healthcare professionals to elevate their level of preparedness when disasters occur.

\section{STUDY LIMITATION}

The study has several limitations due to constraints on research time, place, and methodology design. First, the research aimed to assess the HDP in three Saudi Metropolitan cities (Riyadh, Jeddah, Dammam), which exclude other cities and rural hospitals. Second, the research included three types of hospitals $(\mathrm{MOH}$, other governmental, and university hospitals), but did not attain private, non-governmental hospitals, and primary care centers. Third, the data collection was performed between November 2018 and February 2019 excluding other timeframes. Finally, the research has implemented a convenience sampling which can lead to the under-representation or over-representation of particular groups within the sample and vulnerable to selection bias. 


\section{REFERENCES}

AlHarastani, H. A. M., Alawad, Y. I., Devi, B., Mosqueda, B. G., Tamayo, V., Kyoung, F., ... and Sierra, S. (2020). Emergency and Disaster Preparedness at a Tertiary Medical City. Disaster Medicine and Public Health Preparedness, 1-11. https://doi.org/10.1017/dmp.2020.28

Alrazeeni, D. (2015). Saudi EMS Students' Perception of and Attitudes toward Their Preparedness for Disaster Management. Journal of education and practice, 6(35), 110116.

Al-Shareef, A. S., Alsulimani, L. K., Bojan, H. M., Masri, T. M., Grimes, J. O., Molloy, M. S, Ciottone, G. R. (2017). Evaluation of hospitals' disaster preparedness plans in the holy city of Makkah (Mecca): a cross-sectional observation study. Prehospital and Disaster Medicine, 32(1), 33-45. https://doi.org/10.1017/S1049023X16001229

Althobaity, H. M., Alharthi, R. A., Altowairqi, M. H., Alsufyani, Z. A., Aloufi, N. S., Altowairqi, A. E., ... and Abdel-Moneim, A. S. (2017). Knowledge and awareness of Middle East respiratory syndrome coronavirus among Saudi and NonSaudi Arabian pilgrims. International journal of health sciences, 11(5), 20.

Ardalan, A., Kandi, M., Talebian, M. T., Khankeh, H., Masoumi, G., Mohammadi, R., ... and Mehranamin, S. (2014). Hospitals safety from disasters in IR iran: the results from assessment of 224 hospitals. PLoS currents, 6 .

Bajow, N. A. and Alkhalil, S. M. (2014). Evaluation and analysis of hospital disaster preparedness in Jeddah. Health, 6(19), 2668. https://doi.org/10.4236/health.2014.619306

Baker, O. G., Alamri, A. A. and Aboshaiqah, A. E. (2019). A descriptive study to analyse the disaster preparedness among Saudi nurses through self-regulation survey. Journal of nursing management, 27(7), 1479-1484. https://doi.org/10.1111/jonm.12833

Bayntun, C., Rockenschaub, G. and Murray, V. (2012). Developing a health system approach to disaster management: A qualitative analysis of the core literature to complement the WHO Toolkit for assessing healthsystem capacity for crisis management. PLoS currents, 4. https://doi.org/10.1371/5028b6037259a

CDC (2013). Hospital All-Hazards Self-Assessment Available at: https://www.cdc.gov/phpr/documents/AHPG_FINAL March_2013.pdf

Chaffee, M. W. and Oster, N. S. (2005). Role of Hospitals in Disasters. Prehospital and Disaster Medicine, 20(S2), S138S138. https://doi.org/10.1017/S1049023X00015107

Heidaranlu, E., Ebadi, A., Khankeh, H. R. and Ardalan, A. (2015). Hospital disaster preparedness tools: a systematic review. PLoS currents, 7. https://doi.org/10.1371/currents. dis.7a1ab3c89e4b433292851e349533fd77

Heidaranlu, E., Khankeh, H., Ebadi, A. and Ardalan, A. (2017). An evaluation of non-structural vulnerabilities of hospitals involved in the 2012 east Azerbaijan earthquake. Trauma Mon, 22(2), e28590. https://doi.org/10.5812/ traumamon.28590
Ibrahim, F. A. A. (2014). Nurses knowledge, attitudes, practices and familiarity regarding disaster and emergency preparedness-Saudi Arabia. American Journal of Nursing Science, 3(2), 18-25. https://doi.org/10.11648/j.ajns. 20140302.12

Jenkins, J. L., Kelen, G. D., Sauer, L. M., Fredericksen, K. A. and McCarthy, M. L. (2009). Review of hospital preparedness instruments for National Incident Management System compliance. Disaster medicine and public health preparedness, 3(S1), S83-S89. https://doi.org/10.1097/ DMP.0b013e3181a06c5f

Kaji, A. H., Langford, V. and Lewis, R. J. (2008). Assessing hospital disaster preparedness: a comparison of an on-site survey, directly observed drill performance, and video analysis of teamwork. Annals of emergency medicine, 52(3), 195-201.

https://doi.org/10.1016/j.annemergmed.2007.10.026

Kapur, G. B. and Smith, J. P. (2010). Emergency public health: Preparedness and response. Jones \& Bartlett Publishers.

Mulyasari, F., Inoue, S., Prashar, S., Isayama, K., Basu, M., Srivastava, N. and Shaw, R. (2013). Disaster preparedness: looking through the lens of hospitals in Japan. International Journal of Disaster Risk Science, 4(2), 89-100. https://doi.org/10.1007/s13753-013-0010-1

Nekoie-Moghadam, M., Kurland, L., Moosazadeh, M., Ingrassia, P. L., Della Corte, F. and Djalali, A. (2016). Tools and checklists used for the evaluation of hospital disaster preparedness: a systematic review. Disaster medicine and public health preparedness, 10(5), 781-788. https://doi.org/10.1017/dmp.2016.30

Nelson, C., et al. (2007). Conceptualizing and defining public health emergency preparedness. American Public Health Association, 97(Suppl 1), 9-11. https://doi.org/10.2105/ AJPH.2007.114496

Nofal, A., Alfayyad, I., Khan, A., Al Aseri, Z. and Abu-Shaheen, A. (2018). Knowledge, attitudes, and practices of emergency department staff towards disaster and emergency preparedness at tertiary health care hospital in central Saudi Arabia. Saudi medical journal, 39(11), 1123. https://doi.org/10.15537/smj.2018.11.23026

Shalhoub, A. A. B., Khan, A. A. and Alaska, Y. A. (2017). Evaluation of disaster preparedness for mass casualty incidents in private hospitals in Central Saudi Arabia. Saudi medical journal, 38(3), 302. https://doi.org/10.15537/ smj.2017.3.17483

Sunindijo, R. Y., Lestari, F. and Wijaya, O. (2019). Hospital safety index: assessing the readiness and resiliency of hospitals in Indonesia. Facilities, 38(1/2), 39-51. https://doi.org/10.1108/F-12-2018-0149

White, T., Ariaratnam, S. T. and Michael, J. (2012). Subterranean infrastructure reconnaissance for manmade and natural hazards and disasters. International Journal of Disaster Resilience in the Built Environment, 3(1), 66-86. https://doi.org/10.1108/17595901211201141 
WHO (2012). WHO's hospital safety index and emergency checklist rolled out in Europe, World Health Organization, Geneva, Switzerland. Available at: http://www.euro.who.int /en/health-topics/emergencies/pages/news/news/2012/ 10/whos-hospital-safety-index-and-emergency-checklistrolled-out-in-europe

WHO (2016). Preparing for Disaster - hospital safety index training takes place in Kazakhstan, World Health Organization Geneva, Switzerland. Available at: http://www.euro.who.int/en/countries/kazakhstan/news/n ews/2016/10/preparing-for-disaster-hospital-safetyindex-training-takes-place-in-kazakhstan
WHO. (2009). Hospital preparedness checklist for pandemic influenza: focus on pandemic (H1N1), 2009. Avaiable at: https://www.euro.who.int/en/health-topics/ communicable-diseases/influenza/publications/2009/ hospital-preparedness-checklist-for-pandemic-influenzafocus-on-pandemic-h1n1-2009

World Health Organization. (2019). Hospital safety index: Guide for evaluators. PAHO. 